
\title{
25 Research Suare \\ Relationship of Matrix Stiffness and Cell Morphology in Regulation of Osteo Genesis and Adipogenesis of BMSCs
}

\section{Yutong Guo}

Sichuan University

Yini Qiao

West China Dental Implant Hospital

Shuqi Quan

Sichuan University

Cai Yang

Sichuan University

Juan Li ( $\nabla$ lijuan@scu.edu.cn )

Sichuan University

\section{Research Article}

Keywords: Matrix stiffness, cell morphology, mesenchymal stem cell, osteogenesis, adipogenesis

Posted Date: August 13th, 2021

DOI: https://doi.org/10.21203/rs.3.rs-801324/v1

License: (c) (i) This work is licensed under a Creative Commons Attribution 4.0 International License. Read Full License 


\section{Abstract}

Backgrounds: Both matrix stiffness and cell morphology have been found as important factors directing MSCs (mesenchymal stem cells) differentiation, but cells also spontaneously adapt their morphology under matrix stiffness stimulation. This study aimed to investigate the interplay of cell morphology and matrix stiffness on osteogenesis and adipogenesis of rBMSCs(rat bone BMSCs) on 2D substrates.

Methods and Results: First, we modulated MSCs morphology through different fibronectin (FN) concentrations on tissue culture plates (TCPs). We found FN promoted and osteogenesis of BMSCs while suppressing adipogenesis, mediated by FN-induced F-actin polymerization and cell spreading. Based on these findings, we modulated BMSCs morphology on $0.5 \mathrm{kPa}$ and $32 \mathrm{kPa}$ CytoSoft ${ }^{\circledR}$ plates through FN concentrations. We found BMSCs on $0.5 \mathrm{kPa}$ substrates coated with $300 \mu \mathrm{g} / \mathrm{ml}$ of FN manifested similarly spreading morphology with cells on $32 \mathrm{kPa}$ substrates coated with $100 \mu \mathrm{g} / \mathrm{ml}$ of FN, and cells in both groups dominantly commit osteogenesis. On the other hand, BMSCs on $32 \mathrm{kPa}$ substrates coated with $30 \mu \mathrm{g} / \mathrm{ml}$ of FN manifested similarly restricted morphology with cells in on $0.5 \mathrm{kPa}$ substrates with $100 \mu \mathrm{g} / \mathrm{ml}$ of $\mathrm{FN}$, and in both groups cells mainly commit adipogenesis. Immunofluorescence staining indicated YAP/TAZ mainly located in cytoplasm when cells exhibited restricted morphology on stiff matrices, while exhibiting significant nuclear translocation when cells spread on soft matrices.

Conclusions: Cell morphology overrode effects of matrix stiffness on BMSCs differentiation through more robust regulation of YAP/TAZ. Matrix stiffness depended on cell morphology to regulate osteogenesis and adipogenesis of BMSCs.

\section{Introduction}

Tissue engineering requires precise control of stem cell fate decisions, which remains a challenge in the status quo. For both research and clinical application, bone mesenchymal stem cells (BMSCs) are one of the most widely applied stem cells for their multi-differentiation potential, easy accessibility and little ethical concern [1].

In the last decade, biophysical cues represented by matrix stiffness have been verified to regulate BMSCs differentiation. Anchoring integrin to surrounding matrix, BMSCs exert traction force through cytoskeleton system to perceive their mechanical microenvironment, and matrix stiffness determines the resistance they receive [2]. A rigid substrate of 25-40 kPa produces high resistant force to BMSCs and induces FAs (focal adhesions) assembly and F-actin polymerization that promotes nuclear translocation of transcriptional regulators YAP/TAZ, thereby promoting osteogenesis of BMSCs, while soft substrates of 0.5-5 kPa inhibit the mechanotransduction process above and favor adipogenic differentiation [2-4]. On flat substrates, cytoskeleton dynamics induced by matrix stiffness simultaneously results in cell morphology change: cells spread to large areas with high cytoskeletal tension on rigid substrates while rounding up with reduced cytoskeletal tension on soft substrates [5]. 
However, independent of external mechanical stimulus, cell morphology has also been demonstrated as an important regulator of BMSCs differentiation [6], as under osteogenic-adipogenic coinduction, spreading morphology directly induced BMSCs into osteogenesis while restricted morphology promoted adipogenesis of BMSCs. These findings raised a question that cell morphology whether an accompanied effect or actually the downstream mediator of matrix-stiffness-induced BMSCs differentiation. Recent studies in 3D culture systems showed that matrix stiffness regulated osteogenesis and adipogenesis of BMSCs through adhesive ligand clustering without cell morphology change [7]. As cell mechanobiology has been found distinctive in 3D and 2D systems, and mechanical stimuli in 3D culture system are less controllable and more intertwined than in 2D systems [8], the decoupling of matrix stiffness and cell morphology in the 3D system needs to be reexamined on 2D substrates.

In our study, we investigated the relationship of matrix stiffness and cell morphology in the regulation of osteogenesis and adipogenesis of BMSCs. We applied different concentrations of adhesive protein fibronectin (FN) to modulate cell morphology on substrates of different stiffness to study their interplay on osteogenesis and adipogenesis of BMSCs and YAP/TAZ activity. We believe this study could help elucidate the intricate mechanotransduction process of stem cells and facilitate tissue engineering in the future.

\section{Methods \& Materials}

\subsection{Isolation and culture of rBMSCs}

We isolated rat bone mesenchymal stem cells from 3-week-old male Sprague-Dawley (SD) rats. Isolated cells were cultured in alpha-modified essential medium (a-MEM, Gibco, USA) supplemented with $10 \%$ fetal bovine serum (FBS, Gibco, USA). Non-adherent cells on tissue culture plates were removed. The cells were passaged after reaching $70 \%$ to $80 \%$ confluence and collected with $0.1 \%$ trypsin/EDTA solution. We used the second-passage BMSCs in the following study.

\subsection{Adipogenic-osteogenic co-induction of BMSCs under different concentrations of fibronectin on tissue culture plates}

First, fibronectin (FN) (Corning, USA) was diluted with PBS into a concentration gradient of $0,5,10,20$ $\mu \mathrm{g} / \mathrm{ml}$, and then coated onto tissue culture plates (Corning, USA) before BMSCs were seeded. BMSCs of passage two were plated onto substrates at $5 \times 10^{4}$ cells $/ \mathrm{ml}$ under adipogenic-osteogenic co-induction (Cyagen Biosciences, China).

Second, to investigate the role of the cytoskeleton under effects of FN on BMSCs differentiation, we set three experiment groups: BMSCs plated on TCPs with $0 \mu \mathrm{g} / \mathrm{ml}$ of FN (group FN), BMSCs plated on TCPs with $1 \mu \mathrm{M}$ of cytochalasin D (Apexbio, USA) and without FN (group FN0+CD), and BMSCs plated on TCPs 
with $10 \mu \mathrm{g} / \mathrm{ml}$ of FN and $1 \mu \mathrm{M}$ cytochalasin D (group FN10+CD). BMSCs in all groups were cultured in the complete culture medium of adipogenic-osteogenic co-induction (Cyagen Biosciences, China). Three independent experiments were repeated for each group.

\subsection{Adipogenic-osteogenic co-induction of BMSCs on 0.5 $\mathrm{kPa}$ and $32 \mathrm{kPa}$ CytoSoft ${ }^{\circledR}$ culture plates coated with different concentrations of FN}

The CytoSoft ${ }^{\circledR}$ culture plate (Advanced Biomatrix, USA) is a product covered with a thin layer of uniform and biocompatible silicone with standard elastic moduli. The silicone gels are not susceptible to hydrolysis. We selected $0.5 \mathrm{kPa}$ and $32 \mathrm{kPa}$ CytoSoft ${ }^{\circledR}$ culture plates for experiments after ultraviolet sterilization for 1 hour. First, we coated the standard FN concentration $(100 \mu \mathrm{g} / \mathrm{ml})$ the producer recommended on CytoSoft ${ }^{\circledR}$ plates. After preliminary experiments, we found cells on $32 \mathrm{kPa}$ plates coated with $30 \mu \mathrm{g} / \mathrm{ml}$ of FN exhibited similar morphology with $0.5 \mathrm{kPa}$ plates coated with $100 \mu \mathrm{g} / \mathrm{ml}$ of FN. Also, we found cells on $0.5 \mathrm{kPa}$ plates coated with $300 \mu \mathrm{g} / \mathrm{ml}$ of FN showed similarly restricted morphology with cells on $32 \mathrm{kPa}$ plates coated with $100 \mu \mathrm{g} / \mathrm{ml}$ of FN. Then we investigated BMSCs differentiation and YAP/TAZ activity in these groups.

BMSCs in the a-MEM medium of passage 2 were plated onto $0.5 \mathrm{kPa}$ and $32 \mathrm{kPa}$ CytoSoft ${ }^{\circledR}$ culture plates at densities of $4 \times 10^{4} / \mathrm{ml}$. After 12 hours, cells were exposed to complete medium of adipogenicosteogenic co-induction (Cyagen Biosciences, China) following protocol in previous study [9]. The adipogenic and osteogenic co-induction medium was replenished every 3 days.

\subsection{Osteogenesis and adipogenesis analysis}

After 15 days of co-induction, cells were fixed by $4 \%$ paraformaldehyde and stained with Fast blue and Oil Red $O$ staining (Solarbio, China) which respectively indicated osteogenesis and adipogenesis of BMSCs. Fast Blue RR/naphthol was applied to stain ALP in cells and followed by Milli-Q water (Millipore, USA) rinsing, and then we rinsed cells by $60 \%$ isopropanol and applied Oil Red $O$ to stain fat vacuoles. A Nikon E600 microscope with a color camera (Japan) was used for imaging.

\subsection{Immunofluorescence analysis}

After 15 days of adipogenic/osteogenic co-induction, cells were rinsed with PBS and then fixed with 4\% paraformaldehyde for $30 \mathrm{~min}$. Then cells were permeablized with $0.1 \%$ Triton X-100 (Amresco, USA) for 5 min and then rinsed with PBS 2-3 times. Then cells were incubated with mouse monoclonal YAP (Abnova, Taiwan) and TAZ (Abcam, USA) antibody solutions with 1\% BSA overnight, and removed the second day. Then we rinsed cells with PBS for several times before cells were incubated with rabbit anti 
mouse secondary antibodies (invitrogen, Thermofisher, USA) for several hours. Later, we rinsed cells with PBS for 3-5 times, and then incubated cells with phalloidin-Rhodamine (Yeasen, China) for 30 min to label filamentous actin cytoskeleton, and then cells were rinsed with PBS for 3 times before treated with 5 $\mathrm{mg} / \mathrm{ml}$ 4',6-diamidino-2-phenylindole (DAPI) (Beyotime, China) for $1 \mathrm{~min}$ to stain nuclei. All the samples were rinsed with Milli-Q water before fluorescence microscope observation (Leica, Germany). We applied ImageJ software to measure cell spreading area on immunofluorescence-staining pictures of F-actin.

\subsection{Real-time Rt-PCR}

After 15 days of adipogenic-osteogenic co-induction, total RNA in cells was extracted with Trizol reagent (Invitrogen, USA) and cDNA was synthesized with PrimeScript RT Reagent Kit (TaKaRa, Japan) according to the manufacturer's instructions. The quantitative real-time RT-PCR was then performed using an ABI PRISM 7300 Real-time PCR System (Applied Biosystems, USA). The housekeeping gene, glyceraldehyde 3-phosphate dehydrogenase (GAPDH) was concurrently amplified in each sample as control and was used for normalization. The primers for genes we tested were designed according to NCBI GenBank and are listed in Table 1.

\section{Table 1}

\section{Real-Time RT-PCR primers used in our experiments}

Target gene Primer sequences

RUNX2 F: 5'-cctgaactcagcaccaagtcct-3'

$R: 5^{\prime}$-tcagaggtggcagtgtcatca-3'

OCN

F:5'-accctctctctgctcactctgct-3'

R:5'-gctccaactccattgttgaggtag-3'

CEBP-a F:5'-gccaagaagtcggtggataaga-3'

R:5'-ggcggtcattgtcactggtc-3'

PPARY-2 F: 5'-gtctcacaatgccatcaggttt -3'

R:5'-aggggggtgatatgtttgaact-3'

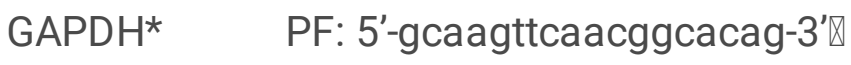

PR: 5'-gccagtagactccacgaca-3'

${ }^{*}$ GAPDH, glyceraldehyde-3-phosphate dehydrogenase

\subsection{Statistical analysis}


Data were presented as mean \pm standard deviation (SD). One way analysis of variance (ANOVA) test and SNK-q test was used to perform statistical analysis using SPSS17.0. Data were collected from three independent experiments with at least 50 cells for each calculation. $P<0.05$ was considered statistically significant.

\section{Results}

\subsection{Increased cell spreading area promoted osteogenesis and inhibited adipogenesis of BMSCs through F-actin assembly}

We modulated cell spreading area through different concentrations of fibronectin (FN) at $5 \times 10^{4} / \mathrm{ml}$ of MSCs on tissue culture plates (TCPs). FN is the adhesive protein exists ubiquitously in ECM that cells attach to integrin and actin cytoskeleton (Kilian et al, 2012), so we explored whether FN could regulate cell morphology and BMSCs differentiation on TCPs, which are stiffer more than several MPas.

For BMSCs differentiation, initially with $0 \mu \mathrm{g} / \mathrm{ml}$ of $\mathrm{FN}$, cells exhibited both fat vacuoles and ALP staining, and as FN concentration increased to $5,10,20 \mu \mathrm{g} / \mathrm{ml}$, more pronounced ALP staining and fewer fat vacuoles were exhibited, indicating BMSCs commit higher extent of osteogenesis and lower adipogenesis (Fig 1A). Also, Rt-PCR illustrated an increase of osteogenic markers (RUNX2 and OCN) and a decrease of adipogenic markers (PPAR- $ү 2$ and CEBP-a) along with higher FN concentration (Fig 1B). These results indicated that larger spreading area directed BMSCs into higher extent of osteogenesis and lower adipogenesis. Meanwhile, immunofluorescence staining indicated that an increase of FN concentration significantly promoted filamentous actin assembly and cell spreading area (Fig 2).

Then, we investigated the underlying role of F-actin in BMSCs differentiation induced by FN. On TCPs with $0 \mu \mathrm{g} / \mathrm{ml}$ of FN at a seeding density of $5 \times 10^{4} / \mathrm{ml}$ (group FNO), after 14 days of adipo-osteo coinduction, cells exhibited both fat vacuoles and ALP deposition (Fig 3). Cytochalasin D is an inhibitor of F-actin polymerization and elongation. In the group that BMSCs cultured with actin polymerization inhibitor cytochalasin $D$ and $0 \mu \mathrm{g} / \mathrm{ml}$ of FN (group FNO+CD), we found cells exhibited mainly fat vacuoles and less ALP deposition (Fig 3A) and PPAR-y2 and CEBP-a exhibited the highest levels in all groups, indicating osteogenesis of BMSCs was inhibited by CD. In the group that BMSCs cultured with $10 \mu \mathrm{g} / \mathrm{ml}$ of $\mathrm{FN}$ and $\mathrm{CD}$ (group FN10+CD), FN rescued osteogenesis of BMSCs and inhibited adipogenesis compared with group FNO+CD, as more ALP and fewer fat vacuoles were exhibited and RUNX2 and OCN mRNA levels were higher than group FNO+CD (Fig 3). These results illustrated that FN increased cell spreading area and promoted osteogenesis of BMSCs through promoting F-actin assembly.

\subsection{Interplay of effects of FN and matrix stiffness on BMSCs differentiation}


Cytosoft ${ }^{\circledR}$ plates with elasticity moduli of $0.5 \mathrm{kPa}$ and $32 \mathrm{kPa}$ were chosen for they respectively resembled rigidity of adipose tissue and pre-calcified bone and have been proven to induce BMSCs into adipogenesis and osteogenesis [10]. As we have verified the effects of FN in regulation of cell spreading area, we seeded BMSCs on soft and stiff substrates coated with different concentrations of FN to modulate cell spreading areas.

First, we found increase of FN concentrations on both $0.5 \mathrm{kPa}$ and $32 \mathrm{kPa}$ substrates induced higher osteogensis and lower adipogenesis of BMSCs (Fig 4). Cells exhibited more pronounced ALP deposition and fewer oil droplets as FN concentration increased from 100 to $300 \mu \mathrm{g} / \mathrm{ml}$ on $0.5 \mathrm{kPa}$ substrates, and from 50 to $100 \mu \mathrm{g} / \mathrm{ml}$ on $32 \mathrm{kPa}$ substrates (Fig 4A). Also, PCR results showed OCN and RUNX2 levels increased while CEBP- $a$ and PPAR- $\gamma$ levels decreased when FN concentrations increased on both substrates (Fig 4B). Meanwhile, immunostaining results showed cells manifested more spreading morphology as FN concentrations increased on both substrates (Fig 5A).

Second, we found when cell spreading area reached similar levels on $0.5 \mathrm{kPa}$ and $32 \mathrm{kPa}$ substrates through different concentrations of FN, cell differentiation of BMSCs didn't manifest significant difference either. We coated $300 \mu \mathrm{g} / \mathrm{ml}$ of $\mathrm{FN}$ on $0.5 \mathrm{kPa}$ substrates(FN300/0.5 kPa group) to achieve similar cell spreading areas with cells on $32 \mathrm{kPa}$ substrates coated with $100 \mu \mathrm{g} / \mathrm{ml}$ of $\mathrm{FN}(\mathrm{FN} 100 / 32 \mathrm{kPa}$ group), and also we coated $30 \mu \mathrm{g} / \mathrm{ml}$ of $\mathrm{FN}$ on $32 \mathrm{kPa}$ (FN30/32 kPa group) to reach similar cell spreading areas with cells on $0.5 \mathrm{kPa}$ substrates coated with $100 \mu \mathrm{g} / \mathrm{ml}$ of $\mathrm{FN}(\mathrm{FN} 100 / 0.5 \mathrm{kPa}$ group), as shown in immunostaining of F-actin in Fig 5A. Then we found when cell spreading areas reached similar levels, cells showed similar extent of osteogenesis and adipogenesis on substrates of different stiffness (Fig 4). Cells in both FN300/0.5 kPa group and FN100/32 kPa group showed mainly ALP staining and few fat vacuoles, indicating BMSCs mainly commit osteogenesis (Fig 4A). PCR results showed consistent results, as OCN and RUNX2 mRNA showed similarly high levels in both groups without significant difference, and CEBP-a and PPAR-y levels showed similarly low levels (Fig 4B). On the contrary, cells in both FN100/0.5 kPa group and FN30/32 kPa group showed little ALP deposition and many fat vacuoles (Fig 4A), and OCN and RUNX2 mRNA showed similarly low levels in both groups without significant difference, and CEBP-a and PPAR- $\gamma$ levels showed similarly high levels (Fig 4B). These results indicated when cell morphology and matrix stiffness exerted antagonistic effects, the former took over. Also, when cell spreading area was set, increase of matrix stiffness failed to exert significant effects on osteogenesis and adipogenesis of BMSCs.

Finally, we found matrix stiffness could exert effects on osteogenesis and adipogenesis of BMSCs only when cell morphology simultaneously adapted. Coated with the same FN concentration of $100 \mu \mathrm{g} / \mathrm{ml}$, matrix stiffening from $0.5 \mathrm{kPa}$ to $32 \mathrm{kPa}$ still prominently promoted osteogenesis and suppressed adipogenesis, as more ALP and fewer fat vacuoles were exhibited (Fig 4A). Also, PCR results indicated significantly higher OCN, RUNX2 and lower CEBP- $a$ and PPAR-y levels as matrix stiffening (Fig 4B). More importantly, cells simultaneously spread to larger areas when matrix stiffness increased, as indicated in immunostaining of F-actin (Fig 5A). 


\subsection{YAP/TAZ activity of BMSCs under regulation of FN and matrix stiffness}

First, we found increase of FN concentration on both $0.5 \mathrm{kPa}$ and $32 \mathrm{kPa}$ substrates induced nuclear translocation of YAP/TAZ of BMSCs. As shown in Fig 5B, YAP/TAZ mainly localized in cytoplasm on 0.5 $\mathrm{kPa}$ substrates coated with $100 \mu \mathrm{g} / \mathrm{ml}$ of FN and on $32 \mathrm{kPa}$ coated with $30 \mu \mathrm{g} / \mathrm{ml}$ of FN, while YAP/TAZ translocated to nuclei on $0.5 \mathrm{kPa}$ substrates coated with $300 \mu \mathrm{g} / \mathrm{ml}$ of FN and on $32 \mathrm{kPa}$ coated with 10 $\mu \mathrm{g} / \mathrm{ml}$ of FN.

Second, when cell spreading area reached similar levels on $0.5 \mathrm{kPa}$ and $32 \mathrm{kPa}$ substrates through respectively $300 \mu \mathrm{g} / \mathrm{ml}$ and $30 \mu \mathrm{g} / \mathrm{ml}$ of FN, YAP/TAZ immunostaining also manifested similar intensity and localization in both nuclei and cytoplasm. As shown in Fig 5B, in both FN300/0.5 kPa group and FN100/32 kPa group, YAP/TAZ mainly localized in nuclei, while in both FN100/0.5 kPa group and FN30/32 kPa group, YAP/TAZ mainly localized in cytoplasm.

Finally, increase of matrix stiffness promoted YAP/TAZ nuclear translocation of BMSCs when cell morphology could simultaneously adapt. As shown in Fig 5B, on substrates coated with $100 \mu \mathrm{g} / \mathrm{ml}$ of FN, increase of matrix stiffness from $0.5 \mathrm{kPa}$ to $32 \mathrm{kPa}$ significantly promoted YAP/TAZ nuclear translocation.

\section{Discussion}

BMSCs adapt their morphology under stimulation of surrounding matrix stiffness through cytoskeleton reorganization, while cell morphology is also an independent factor that induces cytoskeletal dynamics and regulates BMSCs differentiation [11-12]. Also, both cell morphology and matrix stiffness have been found to regulate MSCs differentiation through YAP/TAZ activity [13]. Therefore, the relationship of cell morphology and matrix stiffness was complicated but worth exploring.

First, we verified increased FN concentration promoted BMSCs spreading on TCPs, and osteogenesis of BMSCs was thereby promoted and adipogenesis suppressed. Cytochalasin D is an inhibitor of F-actin assembly, and we found $\mathrm{CD}$ abrogated effects of FN, while higher FN concentration compensated effects of CD. Therefore, FN promoted cell spreading through F-actin polymerization. The process of cell spreading on 2D substrates have been well-documented with FAs assembly and cytoskeleton polymerization and elongation [14]. Therefore, FN induced cell spreading could be regarded as equivalent to natural cell spreading.

Based on these findings, we used various FN concentrations to alter BMSCs morphology on stiff and soft matrix. Again, we verified FN concentrations could regulate cell morphology on Cytosoft ${ }^{\circledR}$ plates. Further, to unravel the relationship of cell morphology and matrix stiffness, we managed to achieve similar cell 
spreading areas on $0.5 \mathrm{kPa}$ and $32 \mathrm{kPa}$ substrates through different FN concentrations. As effects of FN have been equivalent with cell spreading, we could focus on effects of matrix stiffness when cell morphology was set in these situations. Interestingly, we found matrix stiffening from 0.5 to $32 \mathrm{kPa}$ failed to significantly affects MSCs differentiation when cell spreading area was set, and matrix stiffness only exerted significant effects when cell morphology could be simultaneously altered. When cells manifested spreading morphology on both soft and stiff plates, BMSCs commit mainly osteogenesis, while when cells were restricted from spreading regardless on soft and stiff plates, osteogenesis of BMSCs were significantly suppressed while adipogenesis was promoted. These results indicated that matrix stiffness failed to regulate MSCs differentiation when cell morphology didn't exert consistent effects, indicating that matrix stiffness depended on cell morphology to modulate osteogenesis and adipogenesis of BMSCs.

YAP/TAZ are well-documented transcription co-activators that relay biophysical cues and regulate osteogenesis and adipogenesis of BMSCs [16-18]. In our study, we found cell morphology overrode effects of matrix stiffness on YAP/TAZ activity. Cells on soft substrates with spreading morphology induced more prominent YAP/TAZ nuclear translocation than cells on stiff substrates with restricted spreading area, indicating YAP/TAZ was more responsive to cell morphology than matrix stiffness.

Altogether, we found matrix stiffness couldn't exert its effects on BMSCs differentiation without corresponding cell morphology, and cell morphology could be the downstream mechanism of matrixstiffness-induced BMSCs differentiation instead of an accompanying effect through regulation of F-actin assembly and YAP/TAZ activity.

Similar with our study, Harris et al [19] manipulated cell morphology through the Micropattern technique and found stiff matrix didn't promote osteogenesis of BMSCs set with a small area of $1000 \mu \mathrm{m}^{2}$ [19]. Their result indicated matrix stiffness depended on proper cell morphology to regulate BMSCs differentiation. Previous studies found stiff matrix induced cytoskeletal contraction and F-actin elongation [14], while the former alone could be insufficient to significantly affect BMSCs differentiation. In our study, F-actin stress fibers massively assembled in FN-induced BMSCs spreading but didn't significantly assemble in cells on stiff matrix with low FN concentration. Also, Tee et al. found an increase of substrate rigidity barely enhanced cell stiffness when cell morphology was restricted to a small area on Micropattern [20]. Besides, FAs formation and F-actin elongation could be a more important regulator of YAP activity than cytoskeletal contractility, as YAP nuclear localization was reported to be only partially affected by cytoskeletal contraction but predominantly modulated by F-actin assembly $[13,16]$. These studies all implied that F-actin polymerization underlying cell morphology was indispensable for matrix-stiffness-induced BMSCs differentiation.

Recent researches in 3D systems decoupled cell morphology with effects of matrix stiffness on the regulation of BMSCs differentiation, as researchers found clustering of adhesive ligands on adjacent polymer chains and activation of BMSCs-ECM adhesion was sufficient to initiate downstream pathways and affect BMSCs differentiation in the absence of cell spreading $[18,20]$. However, on 2D flat substrates 
where adhesive ligands were coated on the substrate surface, BMSCs could only increase BMSCs-ECM adhesion through higher cell-matrix contact i.e. cell spreading, and this could be part of the reason why matrix stiffness depended on cell spreading to affect BMSCs differentiation in the 2D culture system.

\section{Conclusion}

In conclusion, our research showed that cell morphology was a downstream mediator of the effects of matrix stiffness on osteogenesis and adipogenesis of BMSCs on 2D substrates through regulation of Factin assembly and YAP/TAZ activity. We believe our findings could help elucidate the complicated control system cells constructed in response to mechanical cues and inspire future studies in this field.

\section{Abbreviations}

BMSCs Mesenchymal stem cells

FN fibronectin

\section{Declarations}

Funding

The project was funded by the National Natural Science Foundation of China (31971240).

\section{Competing interests}

The authors declare that they have no competing interests.

\section{Availability of data and materials}

The datasets during and/or analysed during the current study available from the corresponding author on reasonable request.

\section{Code availability}

Not applicable.

\section{Authors' contributions}

Study design: J.L; Experimentation: Y.G, Y.Q; Data Collection \& Analysis: F.J, C.Y, S.Q; Manuscript writing: Y.G. 


\section{Ethics approval and consent to participate}

Not applicable.

\section{Consent for publication}

Not applicable.

\section{Ethical statement}

I certify that the current submission is not previously published or under consideration elsewhere and that all submitted images are in compliance with our image manipulation policy.

All animal procedures performed in this study were in accordance with the Guide for the Care and Use of Laboratory Animals published by the US National Institutes of Health, with approval from Research Ethics Committee of West China Hospital of Stomatology.

\section{Acknowledgements}

Not applicable.

\section{References}

1. Fitzsimmons REB, Mazurek MS, Soos A et al (2018) Mesenchymal Stromal/Stem Cells in Regenerative Medicine and Tissue Engineering, Stem cells international 20188031718

2. Lee J, Abdeen AA, Tang $X$ et al (2016) Matrix-directed adipogenesis and neurogenesis of mesenchymal stem cells derived from adipose tissue and bone marrow, Acta biomaterialia 4246-55

3. Guo M, Pegoraro AF, Mao A et al (2017) Cell volume change through water efflux impacts cell stiffness and stem cell fate. Proc Natl Acad Sci USA 114(41):E8618-E8627

4. Meng Z, Qiu Y, Lin KC et al (2018) RAP2 mediates mechanoresponses of the Hippo pathway. Nature 560(7720):655-660

5. Akhmanova M, Osidak E et al.(2015) Physical, Spatial, and Molecular Aspects of Extracellular Matrix of In Vivo Niches and Artificial Scaffolds Relevant to Stem Cells Research. Stem Cells Int. 2015;2015:167025

6. McBeath R, Pirone DM, Nelson CM et al (2004) Cell shape, cytoskeletal tension, and RhoA regulate stem cell lineage commitment. Developmental cell 6(4):483-495

7. Huebsch N, Lippens E, Lee K et al (2015) Matrix elasticity of void-forming hydrogels controls transplanted-stem-cell-mediated bone formation. Nat Mater 14(12):1269-1277 
8. Bao M, Xie J, Piruska A et al (2017) 3D microniches reveal the importance of cell size and shape, Nature communications 8(1) 1962

9. Peng R, Yao X, Cao B et al (2012) The effect of culture conditions on the adipogenic and osteogenic inductions of mesenchymal stem cells on micropatterned surfaces. Biomaterials 33(26):6008-6019

10. Engler AJ, Sen S, Sweeney HL et al (2006) Matrix elasticity directs stem cell lineage specification. Cell 126(4):677-689

11. Ye K, Cao L, Li S et al (2016) Interplay of Matrix Stiffness and Cell-Cell Contact in Regulating Differentiation of Stem Cells. ACS Appl Mater Interfaces 8(34):21903-21913

12. Kilian KA, Mrksich M (2012) Directing stem cell fate by controlling the affinity and density of ligandreceptor interactions at the biomaterials interface, Angewandte Chemie (International ed. English) 51(20):4891-4895

13. Aragona M, Panciera T, Manfrin A et al (2013) A mechanical checkpoint controls multicellular growth through YAP/TAZ regulation by actin-processing factors. Cell 154(5):1047-1059

14. Ingber DE (2003) Tensegrity I. Cell structure and hierarchical systems biology. Journal of cell science 116(Pt 7):1157-1173

15. Mana-Capelli S, Paramasivam M, Dutta S et al (2014) Angiomotins link F-actin architecture to Hippo pathway signaling. Molecular biology of the cell 25(10):1676-1685

16. Das RK, Gocheva V, Hammink R, Zouani OF, Rowan AE (2016) Stress-stiffening-mediated stem-cell commitment switch in soft responsive hydrogels. Nat Mater 2016 Mar 15(3):318-325.

doi:10.1038/nmat4483. Epub 2015 Nov 30. PMID: 26618883

17. Lee HP, Stowers R, Chaudhuri $O$ (2019) Volume expansion and TRPV4 activation regulate stem cell fate in three-dimensional microenvironments. Nature communications 10(1):529

18. Harris GM, Piroli ME, Jabbarzadeh E (2014) Deconstructing the Effects of Matrix Elasticity and Geometry in Mesenchymal Stem Cell Lineage Commitment. Adv Funct Mater 24(16):2396-2403

19. Tee SY, Fu J, Chen CS, Janmey PA (2011) Cell shape and substrate rigidity both regulate cell stiffness. Biophysical journal 100(5):L25-L27

20. Huebsch N, Arany PR, Mao AS et al (2010), Harnessing traction-mediated manipulation of the cell/matrix interface to control stem-cell fate, Nature materials 9(6)518 - 26.

\section{Figures}




\section{A}

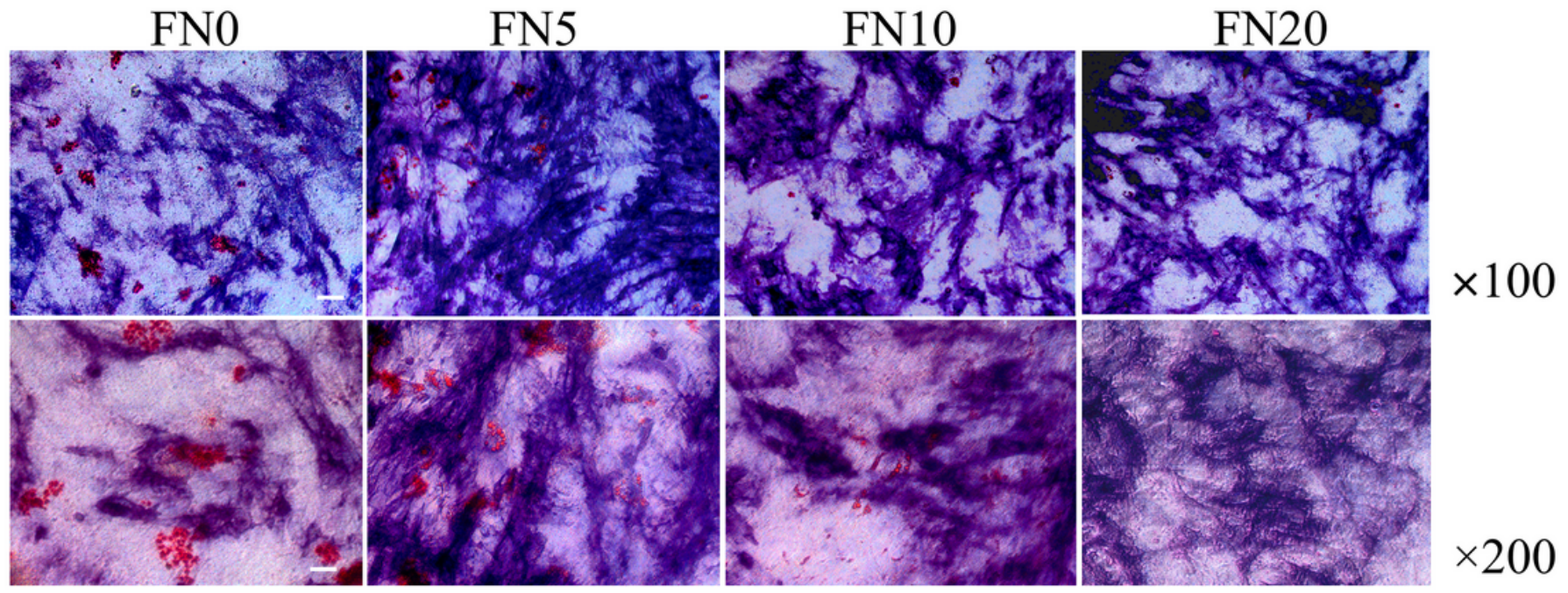

B
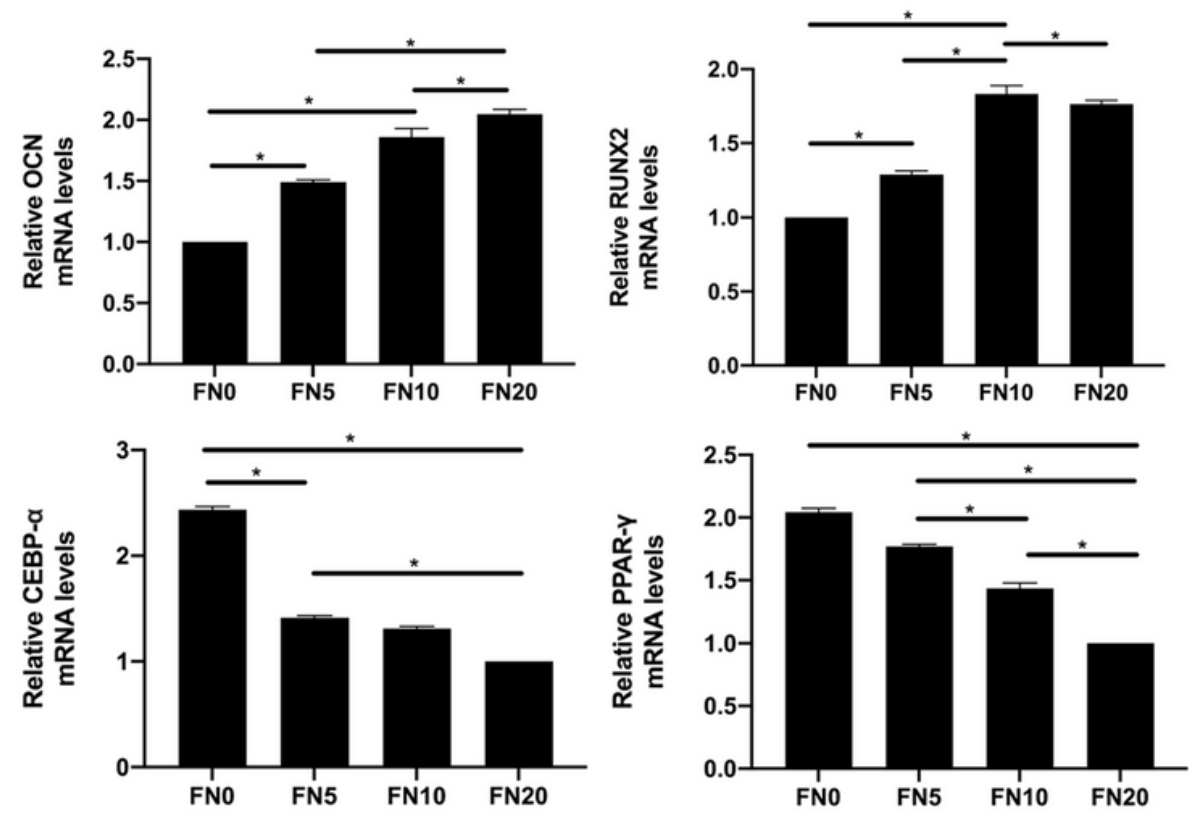

\section{Figure 1}

Osteogenesis and adipogenesis of BMSCs with $0,5,10,20 \mu \mathrm{g} / \mathrm{ml}$ of FN on TCPs. (A) After 15 days of coinduction, cells on TCPs were stained by Fast Blue-Oil Red O concerning ALP (blue) and fat vacuoles (red) $(\times 100, \times 200)$. Scale bars, $50 \mu \mathrm{m}$. Cells exhibited more ALP deposition and fewer fat vacuoles as FN concentration increased. (B) Real time Rt-PCR results of osteogenic markers (OCN, RUNX2) and adipogenic markers (PPAR- $\gamma 2$, CEBP- $a$ ) after 7-day co-induction. Cells showed significantly higher ALP and RUNX2 and lower PPAR- $\gamma 2$ and CEBP- $a$ as FN density increased. ${ }^{*} p<0.05$. 


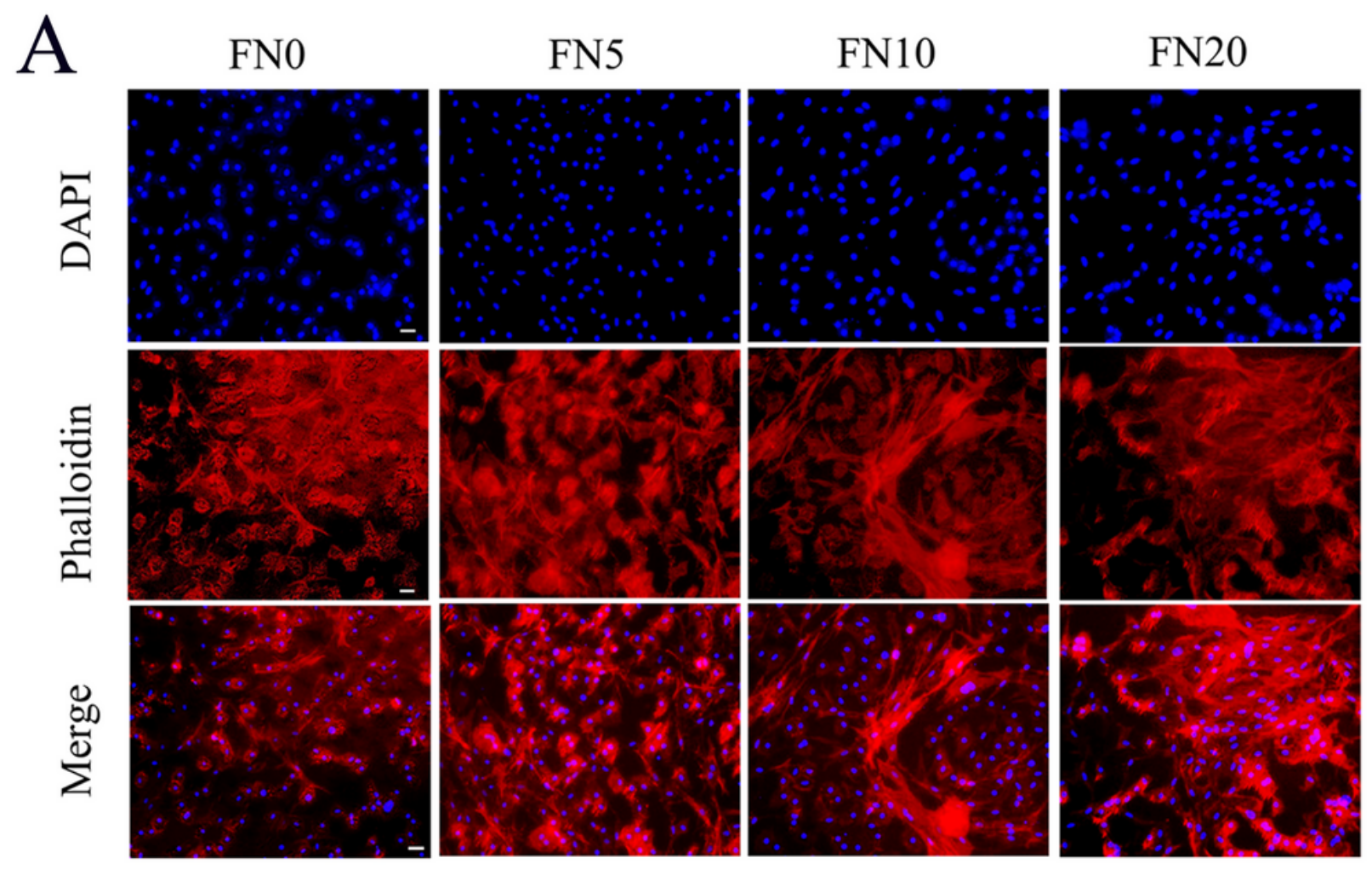

$\mathrm{B}$

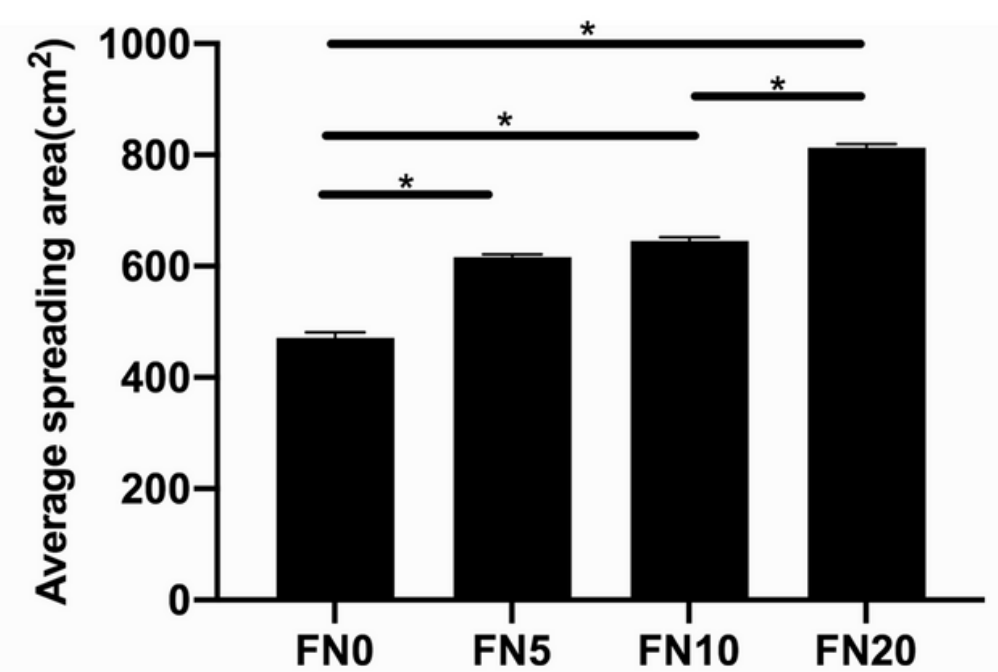

Figure 2

BMSCs morphology and cytoskeleton system with $0,5,10,20 \mu \mathrm{g} / \mathrm{ml}$ of FN. (A) Immunofluorescent images of BMSCs on TCPs after 15 days of adipogenic-osteogenic co-induction with different FN densities. Red: F-actin, blue: nuclei $(\times 100, \times 200)$. Scale bars, $50 \mu \mathrm{m}$. Cells manifested more F-actin stress fibers and spread to a larger area with an increase of FN density. (B) Average spreading area of BMSCs calculated by ImageJ software. The average spreading area of BMSCs was measured by ImageJ software. BMSCs were selected randomly in 10 microscopic fields $(N>50)$. When difficult to isolate individual BMSC at high 
density, we calculated the average projected area as the total area of cell aggregates dividing cell number (measured by the number of cell nucleus). .The cell spreading area significantly increased as FN density rose. ${ }^{*} p<0.05$.
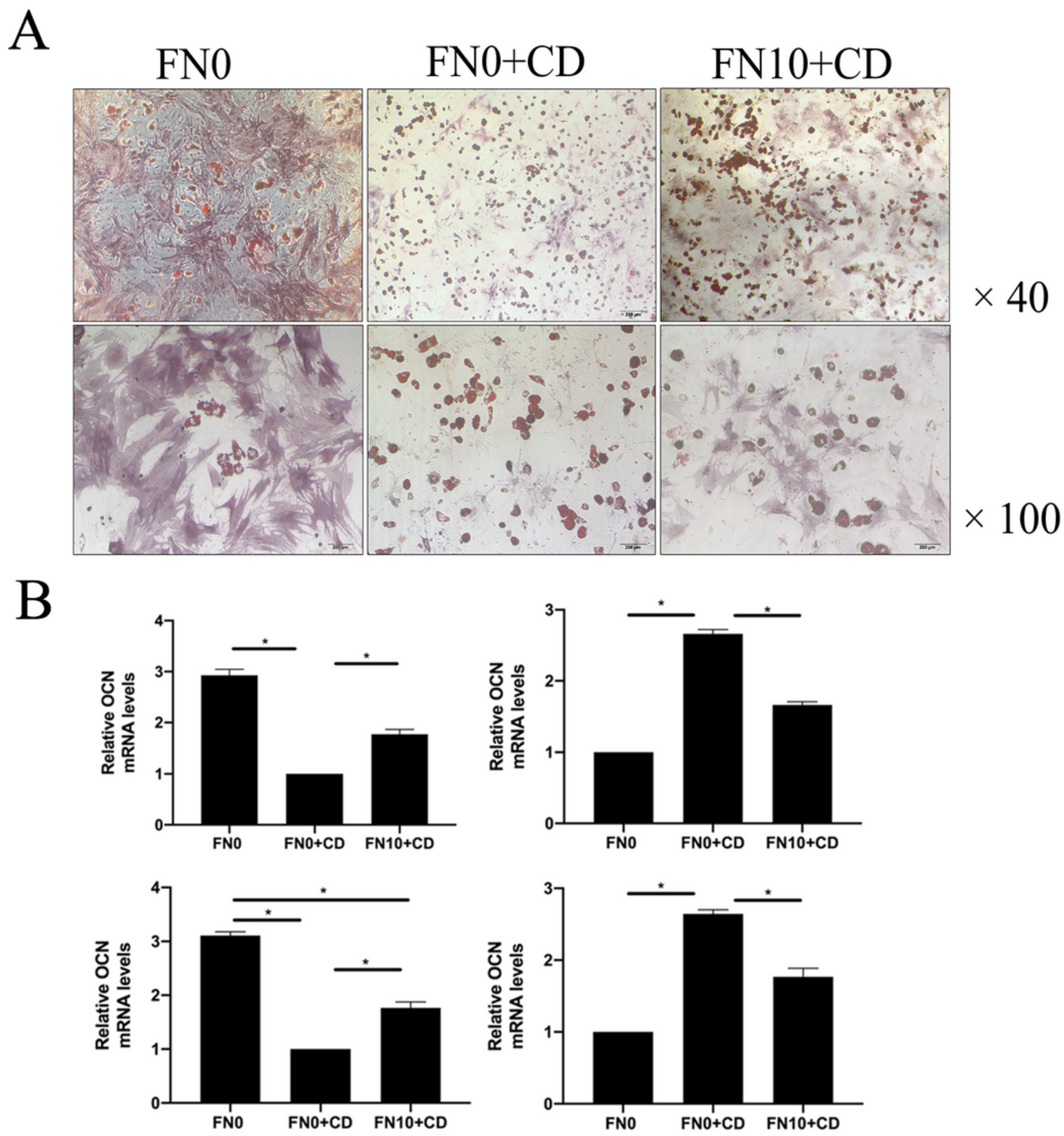

Figure 3

Cytoskeleton dynamics underlying the regulation of FN on BMSCs differentiation. A. After 15 days of adipogenic-osteogenic co-induction, cells at a density of $5 \times 104 / \mathrm{ml}$ on TCPs were stained with Fast BlueOil Red 0 concerning ALP (blue) and fat vacuoles (red) $(\times 40, \times 100$; scale bars, $200 \mu \mathrm{m})$. On substrates 
without FN or other adhesive proteins (group FNO), cells exhibited mainly aggregated fat vacuoles and sparse ALP staining. Cells cultured with $\mathrm{CD}$ and no FN (group FNO+CD) exhibited mainly fat vacuoles and little ALP staining. Cultured with $10 \mu \mathrm{g} / \mathrm{ml} \mathrm{FN}$ and CD (FN10+CD), cells showed pronounced ALP deposition and sparse fat vacuoles. B. OCN, RUNX2, PPAR- $\gamma 2$, CEBP- a mRNA levels of group FN0, group FN0+CD, group FN10+CD after 15 days of osteo-adipo co-induction. ${ }^{*} p<0.05$.

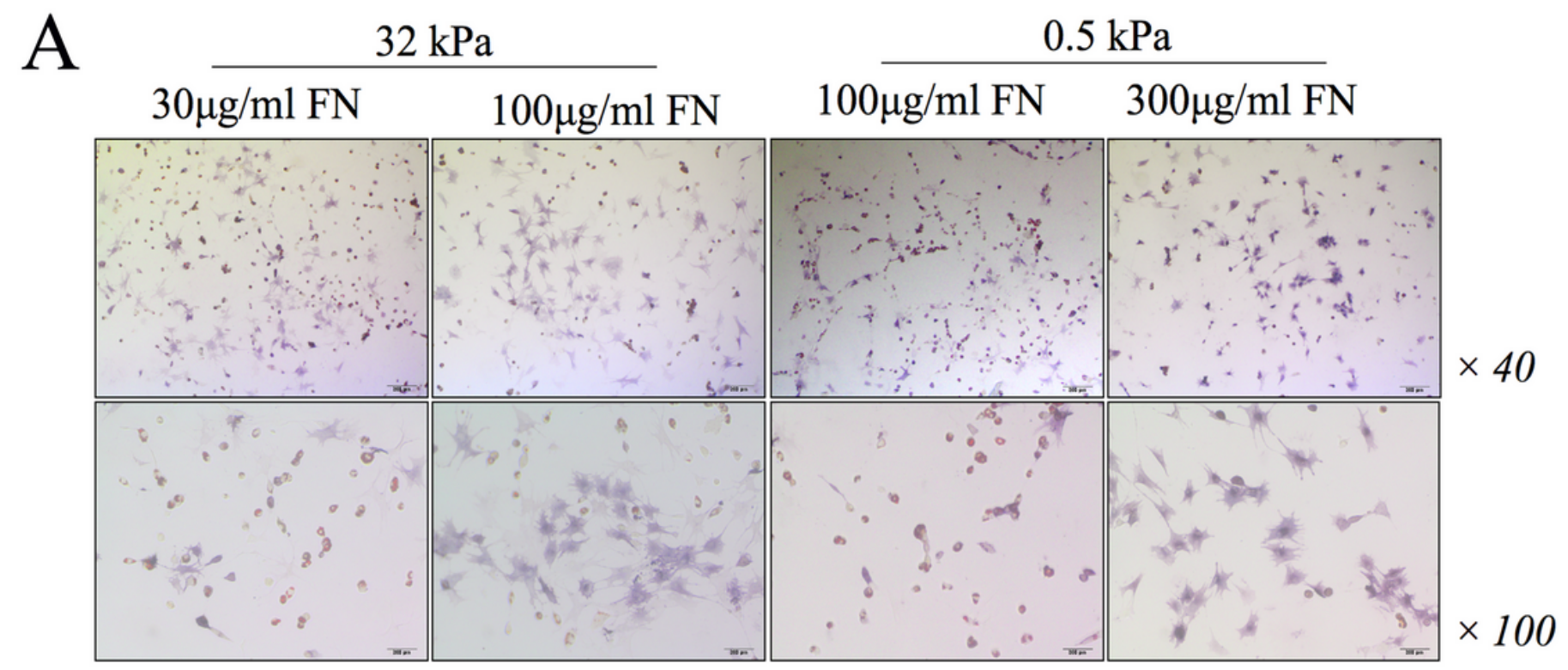

$\mathrm{B}$

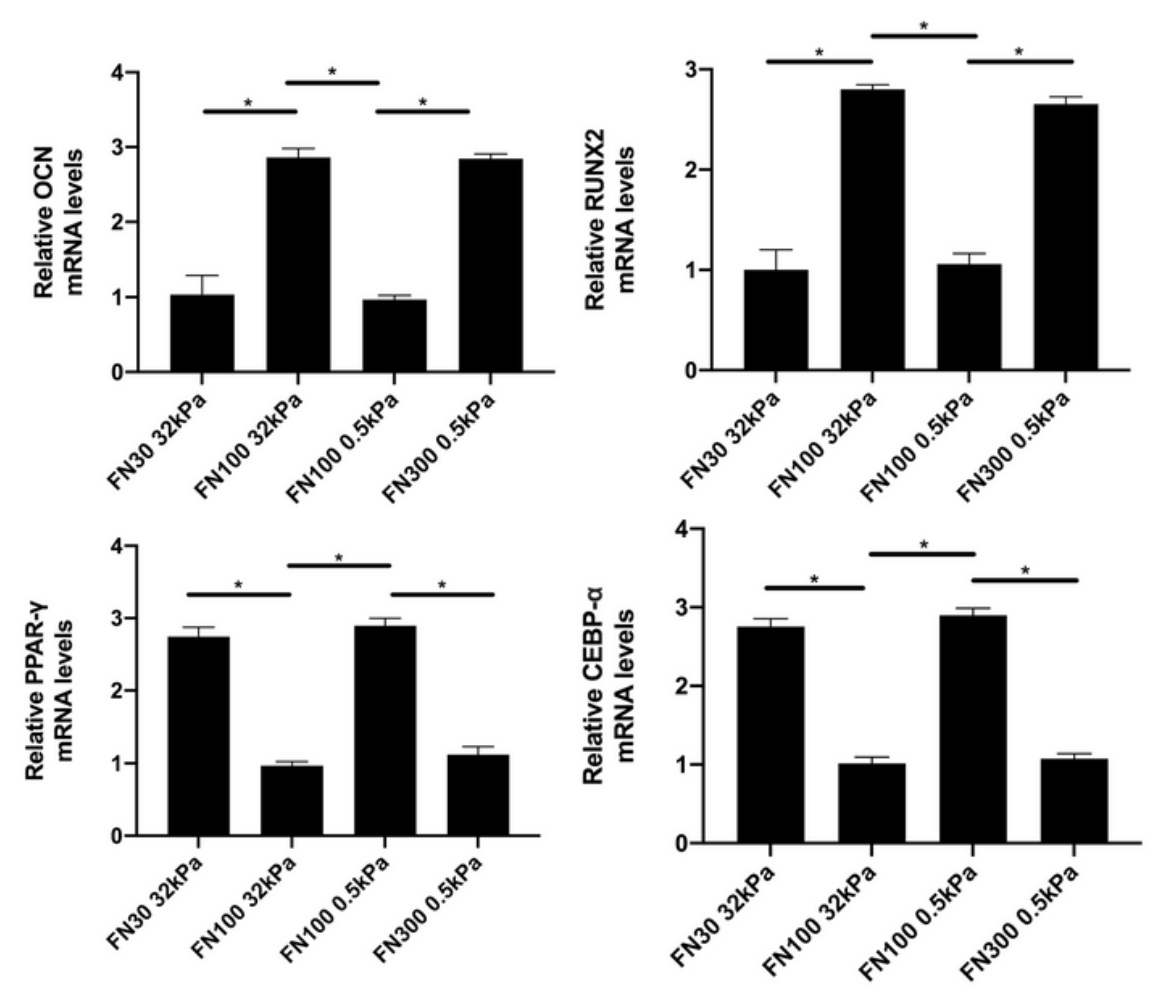

Figure 4 
BMSCs differentiation with different concentrations of $\mathrm{FN}$ on soft and stiff substrates. A. After 15 days of adipogenic-osteogenic co-induction, cells on 0.5 and $32 \mathrm{kPa}$ Cytosoft ${ }^{\circledR}$ plates coated with different FN concentrations were stained with Fast Blue-Oil Red 0 concerning ALP (blue) and fat vacuoles (red) ( $\times 40$, $\times 100$; scale bars, $200 \mu \mathrm{m})$. B. OCN, RUNX2, PPAR- $\gamma 2$, CEBP- a mRNA levels of after 15 days of osteoadipo co-induction. ${ }^{*} \mathrm{p}<0.05$.

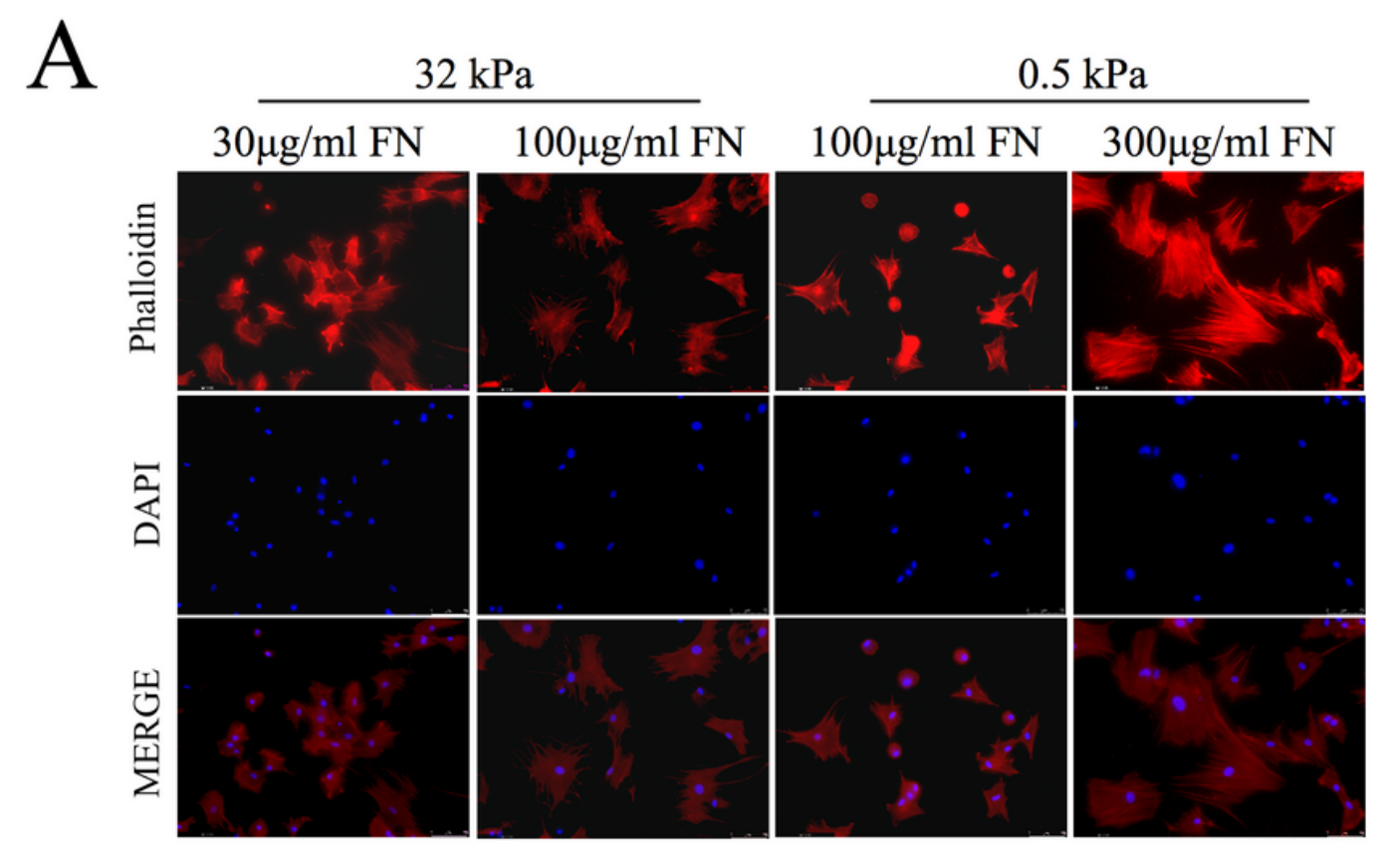

$\mathrm{B}$

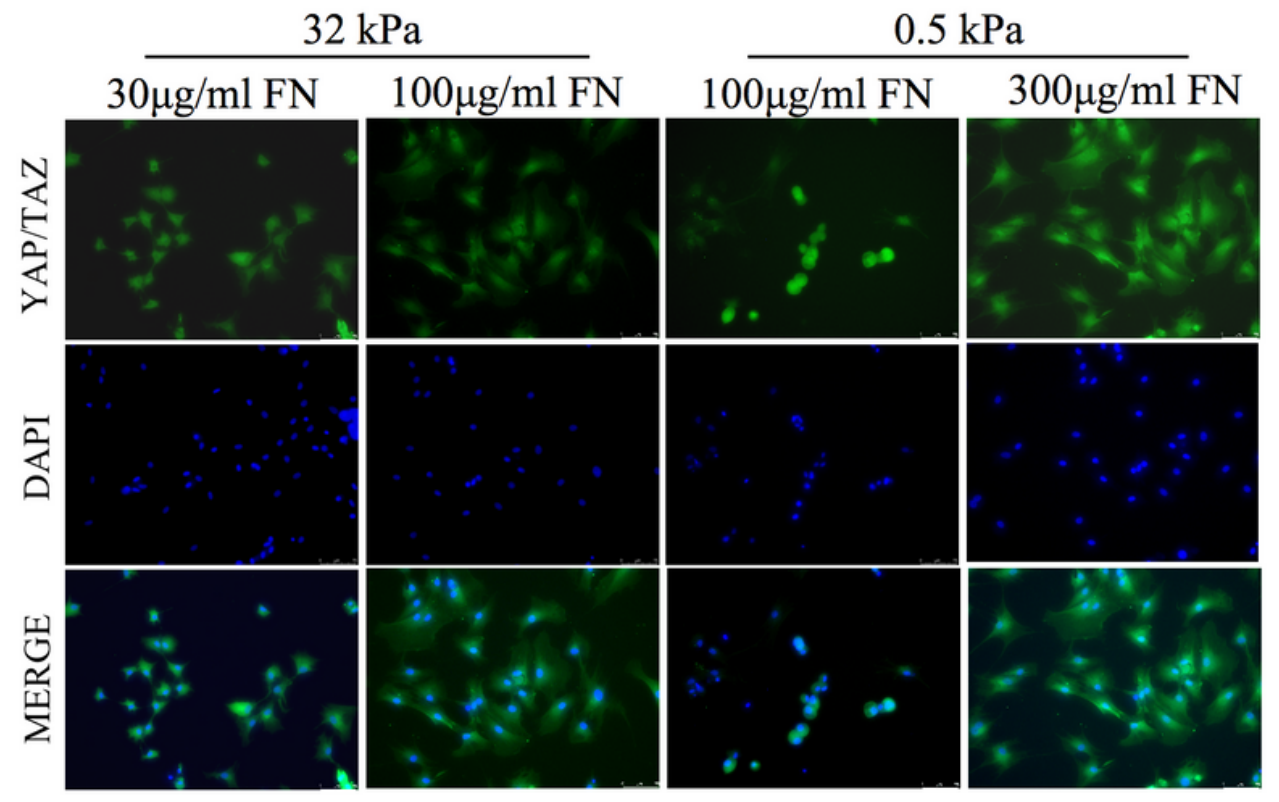

Figure 5 
Immufluorescence staining of F-actin and YAP/TAZ. (A) Immuno-staining of F-actin of BMSCs on $0.5 \mathrm{kPa}$ and $32 \mathrm{kPa}$ substrates after 15 days of adipogenic-osteogenic co-induction. Red: F-actin, blue: nuclei $(\times 100, \times 200)$. Scale bars, $75 \mu \mathrm{m}$. (B) Immuno-staining of YAP/TAZ of BMSCs on $0.5 \mathrm{kPa}$ and $32 \mathrm{kPa}$ substrates. YAP/TAZ manifested nuclear translocation in $0.5 \mathrm{kPa}$ substrates coated with $300 \mu \mathrm{g} / \mathrm{ml}$ of FN and $32 \mathrm{kPa}$ substrates coated with $100 \mu \mathrm{g} / \mathrm{ml}$ of FN, while YAP/TAZ was located in cytoplasm in the other two groups. 